\title{
Unsupervised Pedestrian Re-identification for Loitering Detection
}

\author{
Chung-Hsien Huang, Yi-Ta Wu, and Ming-Yu Shih \\ Advanced Technology Center, Information \& Communications Research Laboratories, \\ Industrial Technology Research Institute, Hsinchiu, Taiwan \\ \{davidchhuang, yitawu, myshih\} aitri.org.tw
}

\begin{abstract}
This paper presents a framework of detecting loitering pedestrians in a video surveillance system. First, to represent pedestrians an appearance feature which contains geometric information and color structure is proposed. After feature extraction, pedestrians are tracked by a proposed Bayesian-based appearance tracker. The tracker takes the advantage of Bayesian decision to associate the detected pedestrians according to their color appearances and spatial location among consecutive frames. The pedestrian's appearance is modeled as a multivariate normal distribution and recorded in a pedestrian database. The database also records time stamps when the pedestrian appears as an appearing history. Therefore, even though the pedestrian leaves and returns to the scene, he/she can still be re-identified as a loitering suspect. However, a critical threshold which determines whether two appearances are associated or not is needed to be set. Thus we propose a method to learn the associating threshold by observing two specific events from on-line video. A 10-minute video about three loitering pedestrians is used to test the proposed system. They are successfully detected and recognized from other passing-by pedestrians.
\end{abstract}

Keywords: Video surveillance, loitering detection, pedestrian re-identification, Bayesian decision, tracking.

\section{Introduction}

With the progress of computer vision, intelligent video surveillance systems have not only been widely investigated as research topics, but also been commercialized, such as ObjectVideo [1], ioimage [2], etc. To date, fundamental research issues of intelligent video surveillance include background subtraction, object detection and tracking, shadow removal, and even object recognition have been discussed extensively. Meanwhile, the researchers draws much attention in recent years to the high-level event detection [3], such as behavior analysis [4], abandon object detection and crowd density analysis. In this study, we focus on analyzing the behavior of loitering and propose a framework to detect loitering pedestrians, even though they leave and return to the area under monitoring.

Loitering refers to the sustained presence of one or more people over a given time period in an area. For example, graffiti offenders spend couple minutes in front of a wall to make their "art", prostitutes or baggers wander in the street corners, drug 
dealers meet clients at bus stations, and people with suicide tendency hesitate at the end of platforms. In [5,6] and most of commercial products, the event of loitering is alarmed by locating and tracking an individual when he/she stays in the field of view (FOV) of the monitoring camera under temporal constrains. In [7], an appearancebased loitering method is developed to re-identify pedestrians and has been applied to detect drug dealers in public transportation areas when considering the leave-andreturn problem.

In this study, a new appearance feature which contains geometric information and color structure is developed to present pedestrians. We also propose a Bayesian-based Appearance Tracker (BAT) to track pedestrians. BAT learns a short-term appearance model to represent a pedestrian via several consecutive frames and then associates the model in the coming frame. The association is performed by Bayesian decision [11] under the consideration of appearances and spatial locations. Meanwhile, the system also maintains a pedestrian database to integrate short-term appearance models as a long-term appearance model. Time stamps of each pedestrian who has visited the scene are also recorded. As a result, the event of loitering can be detected by comparing the time stamps with predefined rules. However, a critical threshold which determines whether two appearances are associated or not is needed to be selected. We thus propose a method to learn this associating threshold by observing specific events from on-line video.

The rest of the paper is organized as follows. Section 2 describes the loitering detection framework. Section 3 reveals the experimental results with a 10-minute testing video and discusses. Section 4 presents the conclusions and future works.

\section{System Description}

Figure 1 shows the flowchart of the proposed loitering detection algorithm. Several preprocessing procedures, such as background subtraction, shadow removal and connected-component labeling, are first performed to an input frame to extract image patches of pedestrian. Color and spatial features to represent the pedestrian are then extracted from each image patch. A proposed Bayesian-based appearance tracker (BAT) algorithm will evaluate those features, called candidates of pedestrian (COP) afterwards in this paper, in the adjacent frames to identify a pedestrian if the COP is stably appeared in the consecutive frames. Once a pedestrian is identified, a time stamp will be recorded into a pedestrian database by either updating the database if the pedestrian has already recorded, or adding a new record if it is a new pedestrian. Last, the event of loitering can be detected by comparing the time stamps of the pedestrian. The details of each module are presented in the following subsections.

\subsection{Preprocessing}

The Gaussian mixture model (GMM)-based background subtraction approach presented by Stauffer and Grimson [8] is a commonly used tool for extracting the moving objects. Basically, it uses couples of Gaussian to model the reasonable variation of the background pixels. Therefore, a pixel will be considered as foreground/moving object if the variation is larger than a threshold. In order to not 
only differentiate proper foreground (moving objects) from static background area, but also remove the casting shadows, the GMSM (Gaussian Mixture Shadow Model) approach proposed by Martel-Brisson and Zaccarin [9] is then applied. GMSM uses the GMM learning ability to build statistical models describing the moving cast shadows and can deal with complex and time-varying illumination.

In order to represent the spatial information of the extracted pedestrain candidates, a blob structure, $\mathbf{b}=\left\{r_{\text {left }}, r_{\text {top }}, r_{\text {right }}, r_{\text {bottom }}\right\}$, is used to record the smallest bounding box containing all the connected foreground pixels in which $r_{\text {left }}, r_{\text {top }}, r_{\text {right }}$ and $r_{\text {bottom }}$ indicate the left, top, right and bottom sides of the blob, respectively. Figure 2 (a) and (b) show a pedestrian and its corresponding foreground blob, respectively.

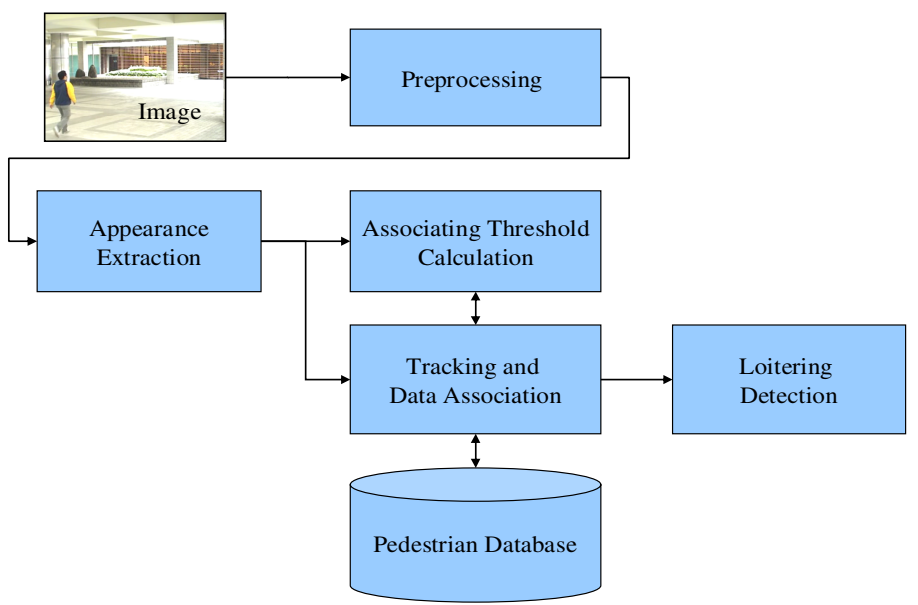

Fig. 1. Flowchart of the proposed loitering detection algorithm

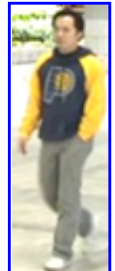

(a)

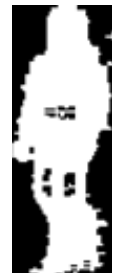

(b)

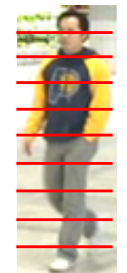

(c)

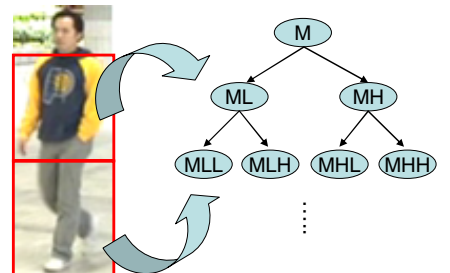

(d)

Fig. 2. Preprocessing and feature extraction of a pedestrian (a) the pedestrian candidate, (b) the GMM-foreground object, (c) Bird et al's approach [7] for feature extraction, and (d) our newly approach for feature extraction

\subsection{Appearance Feature Extraction}

In [7], Bird et al proposed a pedestrian representation method by using color features, as shown in Figure 2(c). They divided a pedestrian candidate into 10 equally spaced horizontal stripes as shown in Figure 2(c), and separately sorted the foreground pixels 
of each strip in R, G, and B channels according to the color intensity. The median in each sorting sequence is then selected as one of the color features of the stripe.

However, Bird et al.'s color feature will have following two problems. First, some strips of the 10 horizontal ones will be sensitive to the noises. For example, the features extracted from the top two strips containing the head portion are not stable since the color feature varies between the skin and hair colors. Second, 10 stripe segmentation is too fine to achieve re-identification if the image size of the pedestrian candidate is small. Therefore, we develop a new feature based on Bird et al.'s approach by adopting the loose segmentation strategy and considering the color structure as shown in Figure 2(d).

For the loose segmentation strategy, we segment a pedestrian candidate into three parts with height ratios of $2: 4: 4$, and the features are extracted from the lower two parts by ignoring the head (top) part. The idea of our newly defined color structure is basically a sampling procedure to sample the histogram. That is, we iteratively segment a parent histogram into two child histograms by a medium intensity of the parent histogram, and collect those medium intensities to form a feature vector. Figure 2(d) shows an example of the color structure. The node of the tree structure contains a medium intensity, $M$, obtained by first sorting the pixels of original histogram, and then selecting the medium value from the sorted list. Obviously, the parent histogram will be separated into two child histograms in which the values in one child histogram will be smaller than the medium value, and the values in another child histogram will be larger than the medium value. We further segment each of the two child histograms into two parts and obtain the medium intensities, $M L$ and $M H$, from the two child histograms, respectively. In this way, we can iteratively segment a parent histogram into two child histograms and collect all the medium intensities from the tree structure to form our feature vector.

As shown in Fig. 2, the feature vector comprises two parts, body and legs. Each part has $3 \times 2^{l-1}$ scalars where 3 represents the $\mathrm{R}, \mathrm{G}$, and $\mathrm{B}$ channels and $l$ is the layer of the tree structure. We set $l=3$. Thus, the feature vector $\mathbf{f}$ of a pedestrian candidate is composed by 24 color scalars as $\mathbf{f}=\left[R_{M L L}^{\text {body }}, G_{M L L}^{\text {body }}, B_{M L L}^{\text {body }}, R_{M L H}^{\text {body }}, \ldots, R_{M H H}^{\text {legs }}, G_{M H H}^{\text {legs }}, B_{M H H}^{\text {legs }}\right]$.

\subsection{Bayesian-Based Appearance Tracker (BAT)}

After the preprocessing at time $t$, a list of pedestrian candidates $\mathbf{C}=\left\{\mathbf{P}_{j}^{t} \mid j=1,2, \ldots, n\right\}$ is obtained for the tracking procedure, where $n$ is the number of candidates in this frame. Obviously, appearance and spatial relationship are important cues to associate candidates between two consecutive frames. We present a Bayesian-based appearance tracker to continuously track a moving object from image sequences. BAT considers the appearance features and spatial locations of two candidates from two adjacent image frames and the association between two candidates will be determined by a Bayesian decision. A sequence of consecutive candidates which have been associated is called a pedestrian's hypothesis $\mathbf{H}=\left\{\mathbf{P}^{1}, \mathbf{P}^{2}, \ldots, \mathbf{P}^{t}, \rho\right\}$ where $\mathbf{P}^{1}$ is the candidate of a pedestrian which appears but associate to none of existing hypotheses, $\mathbf{P}^{2}$ to $\mathbf{P}^{t}$ are consecutively associated candidates, and $\rho$ is called confidence level which indicates 
whether the hypothesis is reliable or not. Our BAT will maintain a list of hypotheses $\mathbf{M}=\left\{\mathbf{H}_{i}^{t} \mid i=1,2, \ldots, m\right\}$ by adding a new candidate if it is not existed in the $\mathbf{M}$ or updating the candidate information if it has already existed in the M. Figure 3 illustrates the flowchart of BAT and the details are described below.

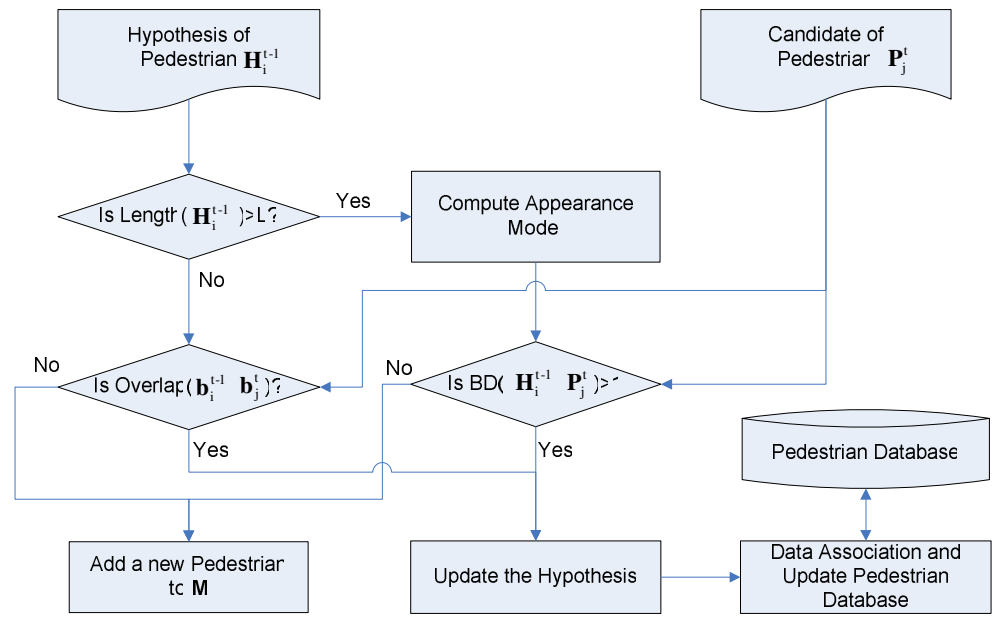

Fig. 3. Flowchart of our BAT

\section{Association by spatial overlap}

For each hypothesis $\mathbf{H}_{i}^{t-1}$ in $\mathbf{M}$, if its trajectory length is shorter than $L$, the hypothesis is at its learning stage. At this stage, the spatial relationship is the only evidence to associate two candidates in different frames. If the blob $\mathbf{b}_{j}^{t}$ and the blob $\mathbf{b}_{i}^{t-1}$ have a large spatial overlapping in image, the candidate $\mathbf{P}_{j}^{t}$ is associated to the hypothesis $\mathbf{H}_{i}^{t-1}$ and then $\mathbf{H}_{i}^{t}$ will be formed by adding $\mathbf{P}_{j}^{t}$ to $\mathbf{H}_{i}^{t-1}$. Note that, $\mathbf{b}_{j}^{t}$ stands for the $j$ th blob in $\mathbf{P}_{j}^{t}$, and $\mathbf{b}_{i}^{t-1}$ stands for the last blob in $\mathbf{P}^{t-1}$ of $\mathbf{H}_{i}^{t-1}$.

\section{Association by appearance and spatial informaiton}

On the other hand, if the trajectory length of $\mathbf{H}_{i}^{t-1}$ is larger than $L$, it means that $\mathbf{H}_{i}^{t-1}$ has been tracked at least $L$ frames, and $\mathbf{H}_{i}^{t-1}$ is already existed in $\mathbf{M}$. Therefore, in this stage, we will merely check whether a candidate $\mathbf{P}_{j}^{t}$ should be associated to $\mathbf{H}_{i}^{t-1}$ by Bayesian decision. The discriminant function is given by Eq. (1):

$$
\begin{aligned}
B D\left(\mathbf{H}_{i}^{t-1}, \mathbf{P}_{j}^{t}\right) & =P\left(C_{\mathbf{H}} \mid \mathbf{P}_{j}^{t}\right) / P\left(\overline{C_{\mathbf{H}}} \mid \mathbf{P}_{j}^{t}\right) \\
& =p\left(C_{\mathbf{H}}\right) p\left(\mathbf{P}_{j}^{t} \mid C_{\mathbf{H}}\right) / p\left(\overline{C_{\mathbf{H}}}\right) p\left(\mathbf{P}_{j}^{t} \mid \overline{C_{\mathbf{H}}}\right)
\end{aligned}
$$

where $C_{\mathbf{H}}$ stands for $\mathbf{P}_{j}^{t}$ is associated to $\mathbf{H}_{i}^{t-1}$, and $\overline{C_{\mathbf{H}}}$ is the complement of $C_{\mathbf{H}}$, i.e. $\mathbf{P}_{j}^{t}$ is not associated to $\mathbf{H}_{i}^{t-1}$. Obviously, if $B D$ is larger than one, there is an association between $\mathbf{P}_{j}^{t}$ and $\mathbf{H}_{i}^{t-1}$. 
The likelihood function $p\left(\mathbf{P}_{j}^{t} \mid C_{\mathbf{H}}\right)$ of class $C_{\mathbf{H}}$ is modeled as a multivariate normal distribution $N\left(\boldsymbol{\mu}, \Sigma^{2}\right)$ as shown in Eq. (2) where $\boldsymbol{\mu}$ and $\sum$ stand for the mean vector and covariance matrix measured by the past $L$ observations (from $\mathbf{f}_{i}^{t-L_{1}}$ to $\mathbf{f}_{i}^{t-1}$ ), respectively.

$$
p\left(\mathbf{P}_{j}^{t} \mid C_{\mathbf{H}}\right)=\frac{1}{\sqrt{\operatorname{det} \sum\left(2 \pi^{d}\right)}} \exp \left(-\frac{1}{2}\left(\mathbf{f}_{j}^{t}-\boldsymbol{\mu}\right)^{T} \Sigma^{-1}\left(\mathbf{f}_{j}^{t}-\boldsymbol{\mu}\right)\right)
$$

Meanwhile, the likelihood function $p\left(\mathbf{P}_{j}^{t} \mid \overline{C_{\mathbf{H}}}\right)$ of class $\overline{C_{\mathbf{H}}}$ is simply modeled as a uniform distribution. Since the prior probabilities should reflect the prior knowledge of $C_{\mathbf{H}}$ and $\overline{C_{\mathbf{H}}}$, the prior probability $p\left(C_{\mathbf{H}}\right)$ is modeled as a similarity metric which inversely proportions to the Euclidian distance between $\mathbf{b}_{j}^{t}$ and $\mathbf{b}_{i}^{t-1}$. Note that, $p\left(\overline{C_{\mathbf{H}}}\right)$ is complement of $p\left(C_{\mathbf{H}}\right)$, i.e. $p\left(\overline{C_{\mathbf{H}}}\right)=1-p\left(C_{\mathbf{H}}\right)$. The measurement of $p\left(C_{\mathbf{H}}\right)$ is shown in Eq. (3).

$$
p\left(C_{\mathbf{H}}\right)=\exp \left(-\frac{D\left(\mathbf{b}_{j}^{t}, \mathbf{b}_{i}^{t-1}\right)}{\sigma^{2}}\right)
$$

where $\sigma$ can be tuned according to the frame rate or the average velocity of walking pedestrian in the scene. As a result, if $B D\left(\mathbf{H}_{i}^{t-1}, \mathbf{P}_{j}^{t}\right) \geq 1$, it shows the strong evidence that the observation $\mathbf{P}_{j}^{t}$ and the hypothesis $\mathbf{H}_{i}^{t-1}$ should be associated; otherwise $\mathbf{P}_{j}^{t}$ and $\mathbf{H}_{i}^{t-1}$ are belonged to different pedestrians.

\section{Hypothesis update}

If $\mathbf{P}_{j}^{t}$ and $\mathbf{H}_{i}^{t-1}$ are associated, $\mathbf{H}_{i}^{t}=\left\{\mathbf{P}_{i}^{1}, \mathbf{P}_{i}^{2}, \ldots, \mathbf{P}_{i}^{t-1}, \mathbf{P}_{j}^{t}\right\}$ is derived by adding $\mathbf{P}_{j}^{t}$ to $\mathbf{H}_{i}^{t-1}$. Meanwhile, the confidence level $\rho_{i}$ is increased by adding a fragment $\Delta \rho$ until it reaches a maximum $\rho_{\max }$. If $\mathbf{H}_{i}^{t-1}$ has not been associated to any candidate of pedestrian $\mathbf{P}^{t}$ at frame $t, \rho_{i}$ is decreased by subtracting $\Delta \rho$. When $\rho_{i}$ is smaller than 0 , we remove $\mathbf{H}_{i}^{t-1}$ from $\mathbf{M}$, the Hypothesis list, since $\mathbf{H}_{i}^{t-1}$ has not been observed for a long time. Any other situation is that if a candidate of pedestrian $\mathbf{P}^{t}$ can not be associated to any hypothesis in $\mathbf{M}$, we add a new hypothesis $\mathbf{H}_{m+1}^{t}=\left\{\mathbf{P}^{t}\right\}$ to $\mathbf{M}$ and set its confidence level $\rho_{m+1}=0$.

\section{Association to pedestrian database}

Taking the advantage of Bayesian decision, each pedestrian is tracked and modeled as a multivariate normal distribution. The distribution is called a short-term appearance model since it is established by observing only $L$ frames. A long-term appearance model, established by integrating short-term appearance models, is utilized to represent each pedestrian and recorded into a pedestrian database. Therefore, even though a visitor left, he/she can be recognized and re-identified when he/she re-enter to the scene. 
If the length of a hypothesis $\mathbf{H}_{i}^{t}$ is a multiple of $L$, a short-term appearance model $V=\{N(\boldsymbol{\mu}, \Sigma),\{s\}\}$ is established by measuring the mean vector $\boldsymbol{\mu}$ and the covariance matrix $\sum$ among the past $L$ observations. Note that, $\{s\}$ is a scalar sequence recording the time stamp when the model is established. As a result, each visitor is represented by his/her appearance model with a time-stamp sequence.

After that, the quotient by dividing the length a hypothesis $\mathbf{H}_{i}^{t}$ by $L$ can be derived, and there exists two situations for the quotient. First, when the quotient is equal to one, i.e. the pedestrian has been tracked in only $L$ frames, two circumstances may be happened. The first circumstance is that the pedestrian $V_{i}$ is a new comer and has not been observed in the past, while the second circumstance is that the $V_{i}$ is returning to the scene. In order to distinguish the two circumstances, $V_{i}$ is compared to all pedestrians listed in the pedestrian database $\left\{V_{k} \mid k=1,2, \ldots, r\right\}$ with their appearances. If the appearance distances between $V_{i}$ and some pedestrians are lower than an associating threshold $\boldsymbol{T}$, then we associate he/she to the closest $V_{k}$. The appearance model is then updated by Eqs. (4)-(6). Otherwise, the pedestrian is regarded as a new comer and added to the database.

$$
\begin{gathered}
\tilde{V}_{k}=\left\{N\left(\tilde{\boldsymbol{\mu}}_{k}, \tilde{\Sigma}_{k}\right),\left\{s_{k}^{1}, s_{k}^{2}, \ldots, s_{k}^{u}, s_{i}^{1}, s_{i}^{2}, \ldots, s_{i}^{v}\right\}\right\} \\
\tilde{\boldsymbol{\mu}}_{k}=\frac{u \cdot \boldsymbol{\mu}_{k}+v \cdot \boldsymbol{\mu}_{i}}{u+v} \\
\tilde{\sigma}_{k}^{2}(x, y)=\frac{u \cdot \sigma_{k}^{2}(x, y)+v \cdot \sigma_{i}^{2}(x, y)}{u+v}
\end{gathered}
$$

where $\sigma^{2}(x, y)$ stands for the element $(x, y)$ in the covariance matrix $\sum$, and $u$ and $v$ are the size of the time-stamp sequences of $V_{k}$ and $V_{i}$, respectively. In this case, $v=1$ because the time-stamp sequence of $V_{i}$ only records a frame index $t$.

Second, when the quotient does not equal one, it means that the pedestrian $V_{i}$ has been tracked more than twice. Since a new pedestrian will be added to the database when he/she appears, definitely there is a pedestrian $V_{k}$ in databased associated to $V_{i}$. $V_{k}$ is then updated by adding $V_{i}$ as given in Eq. (4). Note that, the appearance distance, the distance between two normal distributions, is measured by computing the Kullback-Leibler (KL) divergence [10].

To define a proper associating threshold value $\boldsymbol{T}$ is a critical issue since the threshold is used to determine whether two appearances are associated. Two appearances are marked as "same pedestrian" if the distance is smaller than $\boldsymbol{T}$ and as "different pedestrians" otherwise. We propose a method to learn the associating threshold from the on-line video. This method will be discussed in the next subsection. 


\subsection{Associating Threshold Calculation}

As mentioned in the previous subsection, the threshold to distinguish appearances is the most critical parameter for the problem of pedestrian re-identification. In [7], the threshold value is learnt off-line by Fisher Linear Discriminants (FLD) [11]. However, this approach requires lots of training images to build the FLD classifier and the threshold value can not be used in some conditions such as different lighting conditions. Therefore, we propose a method by considering two specific events to learn the threshold value to handle various conditions. Figure 4 illustrates the examples of these two events and the way to compute the associating threshold.

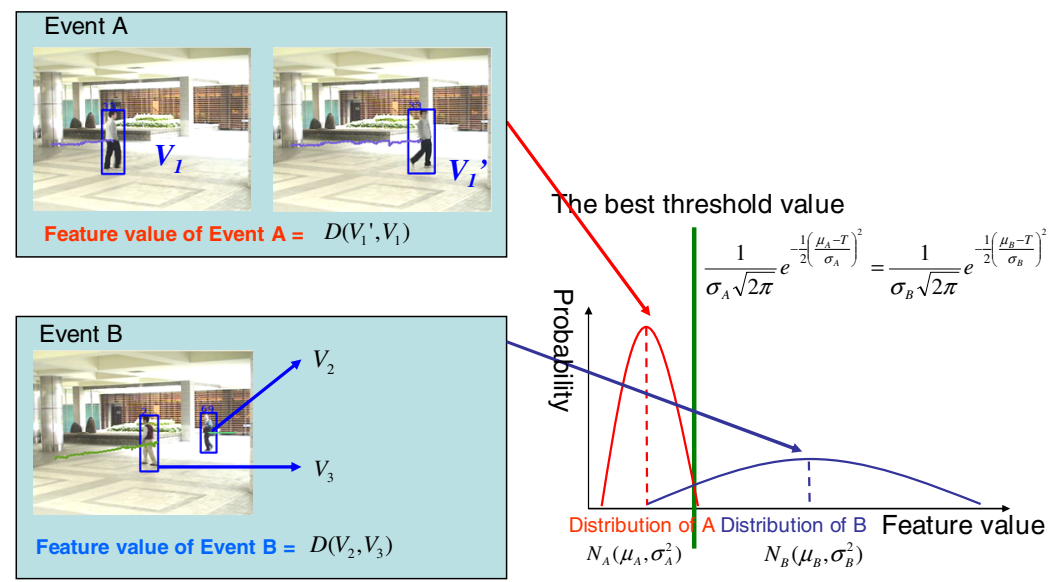

Fig. 4. Examples of two events for calculating the associating threshold

Consider the following two events. In event A, suppose a pedestrian $i$ is stably tracked for $2 L$ frames starting from frame $t$, we can derive two appearances, $V_{1}{ }^{\prime}=A M\left(\mathbf{P}^{t}, \mathbf{P}^{t-1}, \ldots, \mathbf{P}^{t-L+1}\right)$ and $V_{1}=A M\left(\mathbf{P}^{t-L}, \mathbf{P}^{t-L-1}, \ldots, \mathbf{P}^{t-2 L+1}\right)$, from the pedestrian's hypothesis $\mathbf{H}_{i}^{t}$. The feature of event $\mathrm{A}$ is obtained by measuring the appearance distance between $V_{1}$ and $V_{1}{ }^{\prime}$. In event $\mathrm{B}$, suppose there are two visitors $V_{2}$ and $V_{3}$ tracked in the same frame, the feature of event B can be derived by calculating the distance $V_{2}$ and $V_{3}$. After collecting a set of event $\mathrm{A}$ and $\mathrm{B}$, the distributions of their features $N_{A}\left(\mu_{A}, \sigma_{A}^{2}\right)$ and $N_{B}\left(\mu_{B}, \sigma_{B}^{2}\right)$ thus obtained. As a result, the associating threshold $\boldsymbol{T}$ can be determined by solving the following equation.

$$
\frac{1}{\sigma_{A} \sqrt{2 \pi}} e^{-\frac{1}{2}\left(\frac{\mu_{A}-T}{\sigma_{A}}\right)^{2}}=\frac{1}{\sigma_{B} \sqrt{2 \pi}} e^{-\frac{1}{2}\left(\frac{\mu_{B}-T}{\sigma_{B}}\right)^{2}}
$$

\subsection{Loitering Detection}

The definition of loitering is the fundamental of detecting loitering pedestrians. We categorize the loitering events into two class, local loitering and global loitering. The 
local loitering means that the pedestrian keeps loitering in the scene or may just leave for a while. Behaviors of graffiti offenders, street hookers or beggars can be classified into this category. Therefore, if a pedestrian's time stamp sequence is $\left\{s_{1}, s_{2}, \ldots, s_{t}\right\}$, he/she is classified as a locally loitering pedestrian when the following criterion is satisfied:

$$
\frac{s_{t}-s_{j}}{t-j}>\alpha
$$

where

$$
j=\arg \max _{i=1,2, \ldots, i-1}\left(s_{t}-s_{i}\right) \leq t_{i}
$$

The global loitering means that the pedestrian loiters in an area but the camera only covers few part of this area. Therefore, the loitering pedestrian only appears in the video once in a while. Behaviors of drug dealer or pickpocket can be classified into this category. The global loitering is defined when the following criteria are satisfied.

$$
\begin{gathered}
s_{t}-s_{1}>\beta \\
s_{i}-s_{i-1}<\gamma, \quad 1<i \leq t
\end{gathered}
$$

\section{Experimental Results}

To test the proposed system, a 10-min video was captured using Sony DCR-PC 110 DV Handycam camcorder with $320 \times 240$ resolution. The image sequences are obtained with frame rate at 25 frames/sec. For the 10-min video, 16246 frames were captured in which 1685 images of pedestrians have been segmented, accounting for 66 tracking instances tracked BAT. Three of the pedestrians appearing in the video are our colleagues and pretend to be loitering. They left and returned to the scene several times. Other pedestrians are passers who randomly pass by the scene.

\subsection{Discriminability of Color Features}

Figure 5 (a) shows the four pedestrians appearing in our testing video. In order to demonstrate the discriminability in the feature space, we collect 90 patches for each visitor, and display the feature vector in a 3-D Principle Component (PC) space as shown in Fig. 5 (b) by selecting three eigenvectors according to the first three largest eigenvalues after performing the Principle Component Analysis (PCA). Obviously, the clusters of four visitors are well separated at PC space, i.e. the color features are suitable for further high-level pattern analysis.

The comparison of our and Bird's appearance features are provided in Table.1. We generate three independent training and test sets by randomly selecting 15, 25 and 35 patches from the 90 patches for each visitor, and build 3 classifiers in the training set. The classification error rates of three classifiers are $(9 \%, 0.77 \%, 2.27 \%)$ and $(1.3 \%$, $0 \%, 0 \%)$ for Bird's and our approaches. It is clear that our appearance feature outperform Bird's one by reducing the classification error rates. 


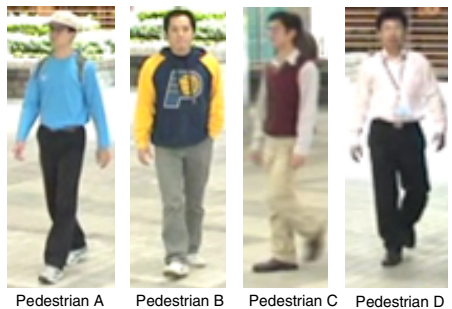

(a)

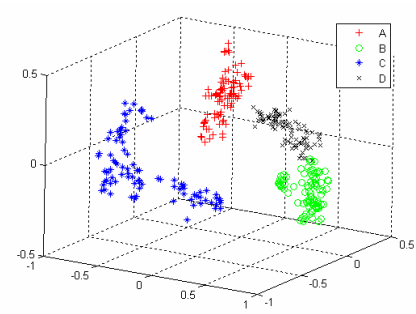

(b)

Fig. 5. Discriminability of the proposed appearance features. (a) Image patches of four visitors (b) Map the color features to a 3-D Principle Component space.

Table 1. Comparison between Bird's appearance feature [7] and the proposed appearance feature

\begin{tabular}{|c|c|c|c|}
\hline Error Rate (\%) & $\mathrm{L}=15$ & $\mathrm{~L}=25$ & $\mathrm{~L}=35$ \\
\hline Bird's appearance feature [7] & $9.00 \%$ & $0.77 \%$ & $2.27 \%$ \\
\hline Proposed appearance feature & $1.30 \%$ & $0.00 \%$ & $0.00 \%$ \\
\hline
\end{tabular}

\subsection{Threshold Selection}

Figure 6 shows an example that the adaptively updated threshold will be converged through the 10-min video. It can be found the threshold varied in the first 3000 frames due to the limited number of positive and negative evidences. After collecting a certain number of evidences, it is not surprised that the threshold is converged.

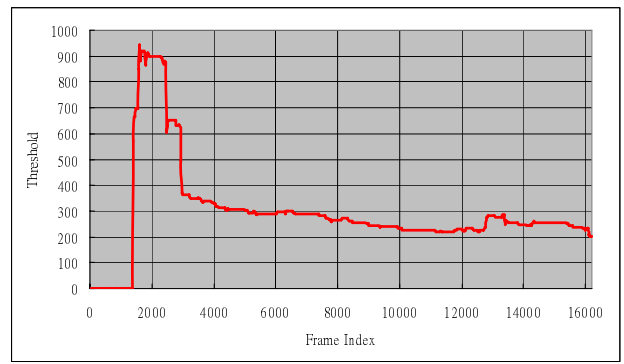

Fig. 6. An example shows the convergence of the updated threshold in the 10-min video

\subsection{Pedestrian Re-identification}

Figure 7 shows the history diagram of LV for the testing video when the threshold $\boldsymbol{T}$ is set to 203.71. Note that the same color spots corresponding to the "same" pedestrian, which were judged by comparing appearance models. The pedestrians A, $\mathrm{B}$, and $\mathrm{C}$, who pretend to be loitering, are tracked and recognized successfully during the video. However, some pedestrians are misclassified as the same person such as (D, D', D' and D', '), and (E, E' and E'). Since only color features are utilized, it is 


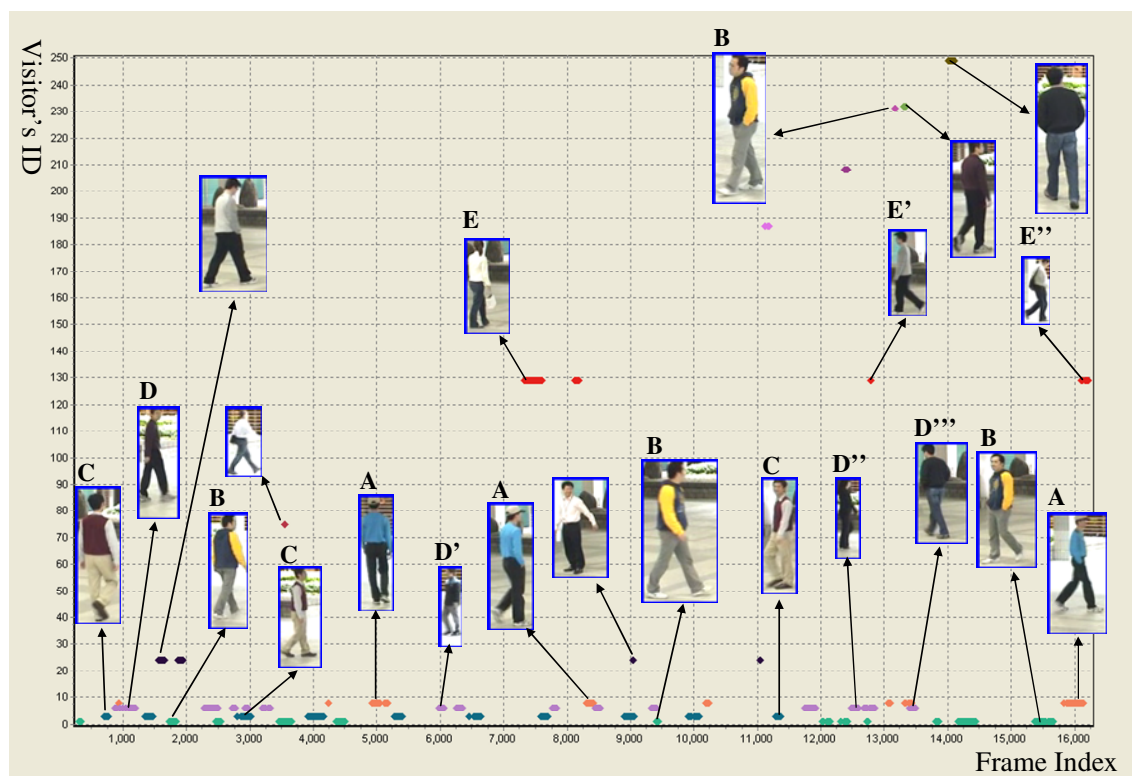

Fig. 7. History diagram of the detected pedestrians of the testing video

Table 2. Sensitivity and specificity of pedestrian re-identification with different approaches

\begin{tabular}{|c|ccc|ccc|}
\hline \hline Method & \multicolumn{3}{|c|}{ Bird's appearance features [7] } & \multicolumn{3}{|c|}{ The proposed method } \\
\hline \hline Pedestrian & $\mathrm{A}$ & $\mathrm{B}$ & $\mathrm{C}$ & $\mathrm{A}$ & $\mathrm{B}$ & $\mathrm{C}$ \\
\hline \hline True positives & 18 & 61 & 50 & 32 & 66 & 63 \\
True negatives & 222 & 170 & 202 & 256 & 222 & 231 \\
False positives & 0 & 20 & 0 & 0 & 0 & 0 \\
False negatives & 13 & 2 & 1 & 6 & 6 & 0 \\
\hline \hline Sensitivity & 0.58 & 0.97 & 0.98 & 0.84 & 0.92 & 1 \\
\hline \hline Specificity & 1 & 0.89 & 1 & 1 & 1 & 1 \\
\hline \hline
\end{tabular}

not surprised that different pedestrians may not be distinguished when they dress similar color clothes.

Accuracy of the classification is then further analyzed. Consider the circumstance of associating the pedestrian A in the database. Suppose the testing pedestrian is A. A true positive (TP) means the testing pedestrian is correctly associated to A. A false negative $(\mathrm{FN})$ occurs when the testing pedestrian is determined as a new pedestrian or associated to others. In contrast, suppose the testing pedestrian is not A. A false positive (FP) occurs when the testing pedestrian is incorrectly associated to $\mathrm{A}$ in the database. A true negative (TN) means the testing pedestrian is associated to other pedestrians or determined as a new pedestrian in the database. The accuracy is evaluated by sensitivity $(=\mathrm{TP} /(\mathrm{TP}+\mathrm{FN}))$ and specificity $(=\mathrm{TN} /(\mathrm{FP}+\mathrm{TN}))$. Table 2 shows the sensitivity and specificity of the loitering pedestrians in the testing video. In the left column, the appearance features were extracted by Bird's method [7] and 
the threshold was selected from the best results of numerous heuristic trials; while the right column shows the result by using the proposed appearance features and the method of calculating the associating threshold. Obviously, the proposed method not only improves the sensitivity and specificity but also avoids heuristic threshold selection, i.e. it can work well without any off-line training.

\section{Conclusion}

This study presents a framework to detect the loitering pedestrian in the video surveillance system. When a pedestrian appears in the FOV, he/she is first tracked by our Bayesian-based appearance tracker (BAT) to form a short-term appearance model of a pedestrian by associate the candidates in the adjacent frames. The association is performed by making a Bayesian decision under the consideration of appearances and spatial locations. Meanwhile, the system also maintains a pedestrian database to record the appearance and time stamps of each pedestrian. The pedestrians recorded can be considered as a long-term appearance model that combines all short-term appearance models of the same pedestrian. When a pedestrian candidate is identified, he/she is then compared to all pedestrians in the database. The pedestrian candidate will be identified as one of the pedestrian in the database and the time stamp will be updated if the difference between two objects are smaller than or equal to a predefined associating threshold. Otherwise, the pedestrian candidate will be added into the database as a new pedestrian. As a result, the behavior of loitering can be detected by comparing the time stamps with user-defined loitering rules. In addition, we propose a method to learn an associating threshold automatically for pedestrian association by observing two specific events from on-line video.

\section{References}

1. ObjectVideo, Inc., http: / /www.objectvideo.com/

2. ioimage Ltd., http: / / www . ioimage.com/

3. Adam, A., Rivlin, E., Shimshoni, I., Reinitz, D.: Robust Real-Time Unusual Event Detection Using Multiple Fixed-Location Monitors. IEEE Trans. Pattern Analysis and Machine Intelligence 30(3), 555-560 (2008)

4. Hsieh, J.-W., Hsu, Y.-T., Liao, H.-Y., Chen, C.-C.: Video-Based Human Movement Analysis and Its Application to Surveillance Systems. IEEE Trans. Multimedia 10(3), 372 $384(2008)$

5. Siebel, N.T., Maybank, S.: Fusion of multiple tracking algorithms for robust people tracking. In: Heyden, A., Sparr, G., Nielsen, M., Johansen, P. (eds.) ECCV 2002. LNCS, vol. 2353, pp. 373-387. Springer, Heidelberg (2002)

6. Black, J., Velastin, S., Boghossian, B.: A Real Time Surveillance System for Metropolitan Railways. In: Proceedings of IEEE Conference on Advanced Video and Signal Based Surveillance, pp. 189-194 (2005)

7. Bird, N.D., Masoud, O., Paapnikolopoulos, P.P., Isaacs, A.: Detection of Loitering Individuals in Public Transportation Areas. IEEE Trans. Intelligent Transportation Systems 6(2), 167-177 (2005) 
8. Stauffer, C., Grimson, W.E.L.: Adaptive Background Mixture Models for Real-time Tracking. Proc. IEEE Comput. Vision Pattern Recognit. 2, 246-252 (1999)

9. Martel-Brisson, N., Zaccarin, A.: Learning and Removing Cast Shadows through a Multidistribution Apprach. IEEE Trans. Pattern Analysis and Machine Intelligence 29(7), 1133-1146 (2007)

10. Kullback, S.: Information Theory and Statistics. Dover Publications, New York (1968)

11. Duda, O.R., Hart, P.E., Stork, D.G.: Pattern Classification. A Wiley-Interscience Publication, Hoboken (2000) 